Bull. Korean Math. Soc. 49 (2012), No. 3, pp. 647-653

http://dx.doi.org/10.4134/BKMS.2012.49.3.647

\title{
A GENERALIZATION OF THE LORENTZIAN SPLITTING THEOREM
}

\author{
JoNG-GUG YUN
}

ABStRACT. In this paper, we obtain a generalized Lorentzian splitting theorem by weakening the assumption of nonnegative Ricci curvature.

\section{Introduction}

In 1982, S. T. Yau [9] conjectured the Lorentzian version of the celebrated Cheeger-Gromoll splitting theorem, which states that if a spacetime $(M, g)$ is timelike geodesically complete and obeys the timelike convergence condition, then it splits as an isometric product $\left(\mathbb{R} \times H,-d t^{2}+h\right)$, provided that $(M, g)$ has a complete timelike line. Much developments have been made to resolve this conjecture (cf. [4], [5]) and Newman [7] at last established a complete proof of the Lorentzian splitting theorem conjectured by Yau using the Lorentzian Busemann functions.

Thereafter, in 1996, G. Galloway and A. Horta [6] presented a general theory of regularity for Lorentzian Busemann functions in future timelike geodesically complete spacetimes and using these results, the authors simplified many aspects of the proof of the Lorentzian splitting theorem.

On the other hand, the assumption of nonnegative Ricci curvature in the Lorentzian splitting theorem (called as the timelike convergence condition in the above) has a special meaning in the context of general relativity. That is to say, it corresponds to the so called strong energy condition and is interpreted as representing the attraction of gravity in some senses.

However, in the inflationary cosmological models, the strong energy condition does not hold during the inflationary phase. Furthermore, contemporary cosmological observations report that the universe is expanding in an accelerating way rather than an decelerating way, which implies that the violation

Received March 15, 2011.

2010 Mathematics Subject Classification. Primary 53C20; Secondary 57S20.

Key words and phrases. splitting theorem, maximum principle.

This research was supported by Basic Science Research Program through the National Research Foundation of Korea(NRF) funded by the Ministry of Education, Science and Technology(2009-0063957). 
of the strong energy condition might be possible in the current state of the universe.

It is therefore of interest to investigate that if the strong energy condition is weakened, then to what extent we can still say about the classical results on the geometric structure of the spacetime. Many of these kinds of research have been made on the existence of singularity of the spacetime by A. Borde, C. Chicone and P. Ehrlich, F. Tipler etc. (cf. [2], [3], [8]).

Motivated by these situations, the purpose of this paper is to establish a generalized Lorentzian splitting theorem under some weakened energy condition by following the lines along the arguments in [6].

Specifically, the weakened energy condition we are considering in this paper can be described as follows:

$\mathrm{C} 1: \liminf \operatorname{in}_{t \rightarrow \infty} \int_{0}^{t} \operatorname{Ric}\left(\sigma^{\prime}(s), \sigma^{\prime}(s)\right) d s \geq 0$ along each timelike geodesic ray $\sigma:[0, \infty) \rightarrow M$.

C2 : There is a constant $K>0$ such that

$$
\limsup _{t \rightarrow \infty} \int_{0}^{t} \max \left\{-\operatorname{Ric}\left(\sigma^{\prime}(s), \sigma^{\prime}(s)\right), 0\right\} d s \leq K
$$

along each timelike geodesic ray $\sigma:[0, \infty) \rightarrow M$.

Here, timelike geodesic ray is a future inextendible timelike geodesic $\sigma$ : $[0, \infty) \rightarrow M$ each segment of which is maximal, that is,

$$
L\left(\left.\sigma\right|_{[a, b]}\right)=d(\sigma(a), \sigma(b)), 0 \leq a<b .
$$

Recalling that the classical strong energy condition says that

$$
\operatorname{Ric}\left(\sigma^{\prime}(s), \sigma^{\prime}(s)\right) \geq 0
$$

for any timelike vector $\sigma^{\prime}(s)$, it is clear to see that the above conditions $\mathrm{C} 1, \mathrm{C} 2$ are automatically satisfied if we assumed the strong energy condition.

Thus the following theorem is a generalization of the Lorentzian splitting theorem in [7] (or in [9]).

Theorem 1.1. Let $(M, g)$ be a timelike geodesically complete spacetime of dimension $n \geq 3$ such that the above conditions C1, C2 are satisfied. Then $(M, g)$ splits isometrically as a product $\left(\mathbb{R} \times H,-d t^{2}+h\right)$, where $(H, h)$ is a complete Riemannian manifold, provided that $(M, g)$ contains a complete timelike line.

\section{A generalized maximum principle}

In order to generalize the Lorentzian splitting theorem in [6], we need to establish a generalized version of maximum principle presented in Proposition 4.4 of $[6]$. 
We first introduce some basic concepts and notations essential to our work as follows (For many notations of which precise descriptions are omitted here (such as $I^{ \pm}(\sigma)$ ), we refer to [6] or the standard reference like [1]).

Let $S$ be a subset of $M$ and $\sigma:[0, \infty) \rightarrow M$ be a future inextendible causal curve emanating from $\sigma(0) \in S$. Then $\sigma$ is called an $S$-ray if $L\left(\left.\sigma\right|_{[0, a]}\right)=$ $d(S, \sigma(a))$ for all $a>0$, where $d(S, q)=\sup \{d(p, q): p \in S\}$ and $d(p, q)$ is the Lorentzian distance from $p$ to $q$.

For any timelike $S$-ray $\sigma$ and $z \in I^{-}(\sigma) \cap I^{+}(S)$, let $z_{n} \rightarrow z$ and put $p_{n}=\sigma\left(r_{n}\right)$, where $r_{n} \rightarrow \infty$. If $\mu_{n}:[0, \infty) \rightarrow M$ is a segment of maximal curve from $z_{n}$ to $p_{n}$, then by the well known results (such as Lemma 2.1 and Lemma 2.4 in [6]), we can construct a ray $\mu\left(=\lim _{n \rightarrow \infty} \mu_{n}\right)$ starting at $z$ which is called a generalized co-ray of $\sigma$.

If every generalized co-ray of $\sigma$ starting at $z$ is timelike, then we say that the generalized timelike co-ray condition holds at $z$.

We also define the Lorentzian Busemann function $b: M \rightarrow[-\infty, \infty]$ associated to a future complete timelike $S$-ray $\gamma:[0, \infty) \rightarrow M$ as follows:

$$
b(x)=\lim _{r \rightarrow \infty} b_{r}(x), \text { where } b_{r}(x)=d(\gamma(0), \gamma(r))-d(x, \gamma(r)) .
$$

For a general theory of regularity for the Lorentzian Busemann functions in the future timelike geodesically complete spacetimes, we refer the reader to [6].

Now we can state our generalized version of maximum principle in [6] as follows.

Proposition 2.1. Let $M$ be a future timelike geodesically complete spacetime which satisfies the conditions C1, C2, and let $\gamma$ be a timelike S-ray. Let $W \subset$ $I^{-}(\gamma) \cap I^{+}(S)$ be an open set on which the generalized timelike co-ray condition holds. Let $\Sigma \subset W$ be a connected smooth spacelike hypersurface with nonpositive mean curvature, $H_{\Sigma} \leq 0$. If the Busemann function $b=b_{\gamma}$ attains a minimum along $\Sigma$, then $b$ is constant along $\Sigma$.

Proof. Proposition 2.1 can be proved along the same lines as in Proposition 4.4 in [6] and we need only to obtain the following modified version of (3-1) in [6].

Lemma 2.2. Assume that the conditions C1, C2 are true and let $\gamma$ be any timelike geodesic ray. Then for any $-\delta<0$ and $t_{0}>0$, there exists $t_{1}\left(>t_{0}\right)$ such that $\Delta d_{r}\left(\gamma\left(t_{1}\right)\right)>-\delta$, where $d_{r}(x)=d(x, \gamma(r))$.

Proof of Lemma 2.2. We first let $\phi(t):=\Delta d_{r}(\gamma(t))$ and obtain the following Riccati inequality (cf. (14.28) in [1]):

$$
\phi^{\prime}(t) \geq \operatorname{Ric}\left(\gamma^{\prime}(t), \gamma^{\prime}(t)\right)+\frac{1}{n-1} \phi^{2}(t) .
$$

We now suppose that the lemma is not true, which implies that there exist $-\delta<0$ and $t_{0}>0$ such that $\phi(t) \leq-\delta$ for all $t>t_{0}$.

We then consider two cases as below.

Case I. $\phi(\tilde{t})=0$ for some $\tilde{t}$ with $0 \leq \tilde{t} \leq t_{0}$. 
First note that by the condition $\mathrm{C} 1$, for any $\epsilon>0$, we can choose $\left\{s_{1}, s_{2}, \ldots\right\}$ with $t_{0}<s_{1}<s_{2}<\cdots \rightarrow \infty$ such that

$$
\int_{0}^{s_{i}} \operatorname{Ric}\left(\gamma^{\prime}(s), \gamma^{\prime}(s)\right) d s \geq-\epsilon
$$

for all $i=1,2, \ldots$.

If we now integrate the above Riccati inequality from $\tilde{t}$ to $s_{i}$, then we obtain

$$
\begin{aligned}
\left(\phi\left(s_{i}\right)=\right) \int_{\tilde{t}}^{s_{i}} \phi^{\prime}(s) d s \geq & \int_{\tilde{t}}^{s_{i}} \operatorname{Ric}\left(\gamma^{\prime}(s), \gamma^{\prime}(s)\right) d s+\frac{1}{n-1} \int_{\tilde{t}}^{s_{i}} \phi^{2}(s) d s \\
= & \int_{0}^{s_{i}} \operatorname{Ric}\left(\gamma^{\prime}(s), \gamma^{\prime}(s)\right) d s-\int_{0}^{\tilde{t}} \operatorname{Ric}\left(\gamma^{\prime}(s), \gamma^{\prime}(s)\right) d s \\
& +\frac{1}{n-1} \int_{\tilde{t}}^{s_{i}} \phi^{2}(s) d s \\
\geq & -\epsilon+\int_{0}^{\tilde{t}} \operatorname{Ric}\left(\gamma^{\prime}(s), \gamma^{\prime}(s)\right) d s+\frac{1}{n-1} \int_{\tilde{t}}^{s_{i}} \phi^{2}(s) d s \\
> & 0
\end{aligned}
$$

for some large $i$, since $\int_{\tilde{t}}^{s_{i}} \phi^{2}(s) d s \rightarrow \infty$ as $i \rightarrow \infty$ (Recall that $\phi(t) \leq-\delta$ for all $\left.t>t_{0}\right)$.

This is a contradiction and the lemma is true for this case.

Case II. $\phi(t) \neq 0$ for any $t>0$.

In this case, we can say

$$
-\frac{1}{\phi^{2}(t)} \geq-\frac{1}{\delta^{2}}
$$

for all $t>t_{0}$.

Now the Riccati inequality for $\phi(t)$ implies that for all $t>t_{0}$

$$
\begin{aligned}
\frac{1}{\phi(t)}=\int_{0}^{t} \frac{\phi^{\prime}(s)}{-\phi^{2}(s)} d s & \leq \int_{0}^{t} \frac{\operatorname{Ric}(s)}{-\phi^{2}(s)} d s-\int_{0}^{t} \frac{1}{n-1} d s \\
& \leq \int_{\{s \mid \operatorname{Ric}(s) \leq 0\} \cap[0, t]} \frac{\operatorname{Ric}(s)}{-\phi^{2}(s)} d s-\frac{t}{n-1} \\
& =\int_{\{s \mid \operatorname{Ric}(s) \leq 0\} \cap\left[0, t_{0}\right]} \frac{\operatorname{Ric}(s)}{-\phi^{2}(s)} d s \\
& +\int_{\{s \mid \operatorname{Ric}(s) \leq 0\} \cap\left[t_{0}, t\right]} \frac{\operatorname{Ric}(s)}{-\phi^{2}(s)} d s-\frac{t}{n-1},
\end{aligned}
$$

where we used $\phi(t) \rightarrow-\infty$ as $t \rightarrow 0$ in the first equality (cf. p. 537 in [1]) and let $\operatorname{Ric}(s)=\operatorname{Ric}\left(\gamma^{\prime}(s), \gamma^{\prime}(s)\right)$. 
If we let $A\left(t_{0}\right)=\int_{\{s \mid \operatorname{Ric}(s) \leq 0\} \cap\left[0, t_{0}\right]} \frac{\operatorname{Ric}(s)}{-\phi^{2}(s)} d s \geq 0$, then the above inequalities turn into the following:

$$
\begin{aligned}
\frac{1}{\phi(t)} & \leq A\left(t_{0}\right)+\int_{\{s \mid \operatorname{Ric}(s) \leq 0\} \cap\left[t_{0}, t\right]} \frac{\operatorname{Ric}(s)}{-\delta^{2}} d s-\frac{t}{n-1} \\
& \leq A\left(t_{0}\right)+\frac{K}{\delta^{2}}-\frac{t}{n-1},
\end{aligned}
$$

where we used the condition $\mathrm{C} 2$ in the second inequality.

Now note that $A\left(t_{0}\right)+\frac{K}{\delta^{2}}-\frac{t}{n-1}$ goes to $-\infty$ as $t \rightarrow \infty$, which implies that $\phi(t) \rightarrow 0$ as $t \rightarrow \infty$.

But this contradicts to the fact that $\phi(t) \leq-\delta$ for all $t>t_{0}$.

Thus the lemma is proved.

Now we can use the above Lemma 2.2 (as a suitable replacement of (3-1) in [6]) to follow the same arguments in the proof of Proposition 4.4 in [6]. In fact, we can now apply the same analysis for $\Delta_{\Sigma} b_{p, t}$ to our case using Lemma 2.2 and complete the proof of Proposition 2.1 (for details, refer to [6]).

\section{Proof of Theorem 1.1}

We can now follow the same line of the proof of the Lorentzian splitting theorem presented in [6] with just a slight modifications.

We first let $\gamma: \mathbb{R} \rightarrow(M, g)$ be any unit speed complete timelike line and define

$$
b_{r}^{+}(x)=r-d(x, \gamma(r)), b_{r}^{-}(x)=r-d(\gamma(-r), x)
$$

and

$$
b^{+}=\lim _{r \rightarrow \infty} b_{r}^{+}, b^{-}=\lim _{r \rightarrow \infty} b_{r}^{-} .
$$

Then by the similar arguments in [6] using our generalized maximum principle (Proposition 2.1), we can show that there exist an open neighborhood $U$ of $\gamma(0)$ in $M$ and a smooth embedded a causal hypersurface $\Sigma$ such that $\Sigma \subseteq$ $\left\{b^{+}=b^{-}=0\right\} \cap U$ (refer to [6] or [1] for details).

For each $q$ in $\Sigma$, we consider the curve $c_{q}: \mathbb{R} \rightarrow(M, g)$ given by $c_{q}(t)=$ $\exp (t N(q))$, where $N(q)$ is a future directed unit normal to $\Sigma$ at $q$. Then it can be shown that $c_{q}(t)$ is a maximal timelike line passing through $q$ which is asymptotic to $\gamma$. Furthermore, these asymptotes $c$ to $\gamma$ turn out to be focal point free to $\Sigma$ at $c(0)$.

If we now consider $E: \mathbb{R} \times \Sigma \rightarrow(M, g)$ defined by $E(t, q)=\exp (t N(q))$, then it can also be shown that $E$ is a diffeomorphism onto its image $U_{1}=E(\mathbb{R} \times \Sigma)$.

Now the Raychaudhuri equation for the mean curvature $H(t)$ of the leaf $\Sigma_{t}=E(\{t\} \times \Sigma)$ says that the following holds.

$$
H^{\prime}(t)=-\operatorname{Ric}(N(c(t)), N(c(t)))-\|\nabla N\|^{2},
$$


where $N=-\nabla b^{+}$is a future directed unit vector field which is normal to $\Sigma_{t}$. If $H\left(t_{0}\right)>0$ (or $H\left(t_{0}\right)<0$ ) for some $t_{0}$, then we can see that $H(t)=-\infty$ at some $t>t_{0}$ (or $t<t_{0}$ ) by the following theorem due to A. Borde [2].

Theorem $3.1([2])$. Let $\gamma(t)$ be a causal geodesic with affine parameter $t$ which is complete for $t \geq t_{0}$. Suppose that for any $\epsilon>0$ there is a $b>0$ such that if $t^{\prime}>t_{0}$ there is an interval $I>t^{\prime}$ of length $\geq b$ with

$$
\int_{t_{0}}^{t} \operatorname{Ric}\left(\gamma^{\prime}(s), \gamma^{\prime}(s)\right) d s \geq-\epsilon \text { for all } t \in I .
$$

Then for any congruence containing $\gamma$ with $H\left(t_{0}\right) \leq 0$ we have either $H(t) \equiv$ 0 for all $t \geq t_{0}$ or $H \rightarrow-\infty$ at some $t>t_{0}$. Here $H$ is the expansion of the congruence defined by $H=\operatorname{div}\left(D \gamma^{\prime}\right)$ and it satisfies the Raychaudhuri equation.

But this contradicts to the fact that the asymptotes $c$ to $\gamma$ are focal free to $\Sigma$. Thus we have $H(t) \equiv 0$ for all $t$ and from the above Raychaudhuri equation, we know that

$$
0 \leq \liminf _{t \rightarrow \infty} \int_{0}^{t} \operatorname{Ric}(N(c(s)), N(c(s))) d s=-\limsup _{t \rightarrow \infty} \int_{0}^{t}\|\nabla N\|^{2} d s,
$$

which implies that

$$
\limsup _{t \rightarrow \infty} \int_{0}^{t}\|\nabla N\|^{2} d s \leq 0 .
$$

This is possible only if we have $\|\nabla N\|^{2} \equiv 0$, that is $\nabla N \equiv 0$. Thus $N$ must be parallel in $U_{1}=E(\mathbb{R} \times \Sigma)$ and this implies that $E$ is an isometry of $(\mathbb{R} \times$ $\left.\Sigma,-d t^{2} \oplus h\right)$ and $\left(U_{1},\left.g\right|_{U_{1}}\right)$.

It can be further shown that this local splitting may be extended to a global splitting by the standard arguments in [1] or [4]. So, we complete our proof of Theorem 1.1 .

\section{References}

[1] J. Beem, P. Ehrlich, and K. Easley, Global Lorentzian Geometry, 2nd ed., Marcel Dekker, New York, 1996

[2] A. Borde, Geodesic focusing, energy conditions and singularities, Classical Quantum Gravity 4 (1987), no. 2, 343-356.

[3] C. Chicone and P. Ehrlich, Line integration of Ricci curvature and conjugate points in Lorentzian and Riemannian manifolds, Manuscripta Math. 31 (1980), no. 1-3, 297-316.

[4] J.-H. Eschenburg, The splitting theorem for space-times with strong energy condition, J. Differential Geom. 27 (1988), no. 3, 477-491.

[5] G. Galloway, The Lorentzian splitting theorem without the completeness assumption, J. Differential Geom. 29 (1989), no. 2, 373-387.

[6] G. Galloway and A. Horta, Regularity of Lorentzian Busemann Functions, Trans. Amer. Math. Soc. 348 (1996), no. 5, 2063-2084.

[7] R. P. A. C. Newman, A proof of the splitting conjecture of S.-T. Yau, J. Differential Geom. 31 (1990), no. 1, 163-184.

[8] F. J. Tipler, Singularities and causality violation, Ann. Physics 108 (1977), no. 1, 1-36.

[9] S.-T. Yau, Problem section, Seminar on Differential Geometry, pp. 669-706, Ann. of Math. Stud., 102, Princeton Univ. Press, Princeton, N.J., 1982. 
Department of Mathematics Education

Korea National University of Education

Chungbuk 363-791, Korea

E-mail address: jgyun69@knue.ac.kr 